\title{
Factores Socioculturales Que Interfieren En La Realización Del Papanicolaou En Mujeres Indígenas Mexicanas
}

\author{
Francisca Velasco Zapata \\ Estudiante de la Licenciatura en Enfermería. División Académica \\ Multidisciplinaria de los Ríos, \\ Universidad Juárez Autónoma de Tabasco, México. \\ Armando Miranda de la Cruz \\ Lorena Magaña-Olán \\ Janett Marina García Hernández. \\ Jairo David Contrera Madrigal
}

Profesores Investigadores de la División Académica Multidisciplinaria de los

Ríos, Universidad Juárez Autónoma de Tabasco, México.

Doi: 10.19044/esj.2018.v14n6p69 URL:http://dx.doi.org/10.19044/esj.2018.v14n6p69

\begin{abstract}
Introduction: Papanicolaou test was introduced in 1943 by the Dr. George Papanicolaou as an examination of early detection for the Cancer Cérvico Uterino (CACU), it is the most frequent test of detection in women who do not have symptoms. It is the most common second type of cancer in women, of the different types of cancer that they affect the women in reproductive present age in developing countries, rural populations and indigenous zones. I target: to identify the sociocultural factors that they interfere in the accomplishment of the Papanicolaou in wives of a community indigenous to Saint Thomas of Tenosique municipality of Pino Suárez, Tabasco. Methodology: Quantitative descriptive, market investigation across the sampling not probabilistic for incidental convenience with one $n=23$ women with ages between 25 and 35 alive years sexual activates. Results: Results: it predominated over the item of age between 29 and 35 years; the level of education that prevails is the primary one, for the marital status the majority is in free union and up to the moment it has had 2 sexual pairs in his life; the beginning of sexual active life that prevails is 16 to 20 years, and the Papanicolaou has never been realized by motives of lack of interest, by the influence of the pair and for not knowing the purpose of there being realized the procedure of periodic form. Conclusion: This study demonstrates that the women have an indifferent attitude towards Papanicolaou capture; since 83.3
\end{abstract}


$\%$ of the women presents this attitude, of equal way $70.8 \%$ agrees very in that there is important the opinion of his pair the test to take.

Keywords: Papanicolaou test, Cervical neoplasms, Culture

\section{Resumen:}

Introducción: La prueba de Papanicolaou fue introducida en 1943 por el Dr. George Papanicolaou como un examen de detección temprana para el Cáncer Cérvico Uterino (CACU), es la prueba más frecuente de detección en mujeres que no tienen síntomas. Es el segundo tipo de cáncer más común en mujeres, de los diferentes tipos de cáncer que afectan a las mujeres en edad reproductiva presente en países en desarrollo, poblaciones rurales y zonas indígenas. Objetivo: identificar los factores socioculturales que interfieren en la realización del Papanicolaou en mujeres de una comunidad indígena de Santo Tomas del municipio de Tenosique de Pino Suarez, Tabasco. Metodología: investigación cuantitativa descriptiva, prospectiva a través del muestreo no probabilístico por conveniencia incidental con una $n=23$ mujeres con edades entre 25 y 35 años con vida sexual activa. Resultados: predominó el rubro de edad entre los 29 y 35 años; el nivel de escolaridad que predomina es la primaria, para el estado civil la mayoría está en unión libre y hasta el momento ha tenido 2 parejas sexuales en su vida; el inicio de vida sexual activa que predomina es de 16 a 20 años, y nunca se han realizado el Papanicolaou por motivos de falta de interés, por la influencia de la pareja y por no conocer la finalidad de realizarse el procedimiento de forma periódica. Conclusión: Este estudio demuestra que las mujeres tienen una actitud indiferente hacia la toma de Papanicolaou; ya que el $83.3 \%$ de las mujeres presenta esta actitud, de igual manera el $70.8 \%$ está muy de acuerdo en que es importante la opinión de su pareja para tomarse la prueba.

Palabras claves: Prueba de Papanicolaou, Neoplasias del Cuello Uterino, Cultura

\section{Introduccion}

De acuerdo a la NOM-014-SSA-1994 para la prevención, tratamiento, y control del cáncer del cuello del útero (Secretaria de Salud [SS], 1998), constituye una prioridad en países en desarrollo como México, los cambios de estilo de vida producidos en los últimos 50 años han modificado los patrones de enfermar y morir, determinando un gran reto para los servicios de salud. Por tal razón, los Sistemas de Salud deberán responder a este cambio y adoptar nuevas estrategias ante la inestable morbimortalidad actual, entre ellas el cáncer, no solo por atender la creciente demanda de los servicios por padecimientos neoplásicos, además se debe cambiar el enfoque a través del 
fortalecimiento de la prevención de enfermedades en el primer nivel de atención a la salud.

En las últimas décadas desde 1992 a la actualidad se ha incrementado la presencia de cáncer del cuello uterino como causa de morbi-mortalidad; por lo tanto el Sistema Nacional de Salud en México, debe adecuarse fortaleciendo estrategias como: la coordinación de los sectores públicos y privados para afrontar estos padecimientos con mayor eficiencia y efectividad. Será también importante una participación activa de la comunidad en la solución de estas enfermedades. Los beneficios que se esperan obtener de la aplicación de la Norma Oficial Mexicana del cáncer Cérvico Uterino implican la reducción de la mortalidad y morbilidad así como de las complicaciones que este padecimiento genera.

Según American Society of Clinical Oncology (ASCO, 2016) la prueba de Papanicolaou, también denominada citología vaginal, es el método más frecuente de detección del cáncer de cuello uterino en las mujeres que no tienen síntomas de cáncer. Por lo general, se realiza durante el control ginecológico de la mujer en edad reproductiva. La prueba de Papanicolaou consiste en obtener una muestra de células del cuello uterino; algunas de las células obtenidas del cuello uterino durante la prueba de Papanicolaou también pueden examinarse para detectar el Virus del Papiloma Humano (VPH, también denominado Virus del Papiloma Humano (VPH), siendo este un factor de riesgo de cáncer de cuello uterino, el cual se transmite mayormente de una persona a otra durante las relaciones sexuales; existen distintos tipos o cepas del VPH, algunas cepas se vinculan más estrechamente con determinados tipos de cáncer del cuello uterino.

Por lo tanto se da por entendido que el no acudir a una cita con un médico especializado para realizarse este procedimiento de detección, puede llegar a ser muy perjudicial para la salud debido la presencia de una Infección de Trasmisión Sexual o una enfermedad del tipo cancerígeno, pero cada individuo tiene diferentes formas de pensar y de ver la vida; dependiendo lo anterior del nivel educativo y socioeconómico de las mujeres. Es decir en cada persona hay una serie de factores que influyen en su toma de decisiones y por ello la importancia de saber cuáles son estos factores y como intervienen en las personas.

Los estudios socioculturales siempre implican vinculación con conceptos y términos tales como ideología, comunicación, etnicidad, clases sociales, estructuras de pensamiento, género, nacionalidad, medios de producción y muchos otros que sirven para comprender los elementos únicos de cada comunidad, sociedad y etnia. Se utiliza el término sociocultural para hacer referencia a cualquier proceso o fenómeno relacionado con los aspectos sociales y culturales de una comunidad o sociedad. De tal modo, que un elemento sociocultural tendrá que ver exclusivamente con las relaciones 
humanas que puedan servir para organizar la vida comunitaria como para darle significado a la misma (Bembibre 2009 ).

En un estudio realizado en San Cristóbal de Las Casas, Chiapas, México sobre las posibilidades sociales de prevención de la infección VPH y Cáncer Cervicouterino (CACU), se obtuvieron resultados en diferentes esferas donde la segunda esfera tenemos las relaciones de pareja. A este respecto manifiestan las negativas de sus parejas varones para "aceptar protegerse" y que éstos no se "cuidan" cuando tienen relaciones sexuales con otras mujeres, lo cual hace evidencia de prácticas como la infidelidad, mala comunicación y algunas conductas "machistas", como prohibir a sus parejas que acudan a revisión ginecológica porque "creen que la intimidad es de su propiedad".

En la tercera esfera se encuentra el nivel social, donde identificamos dos factores, el educativo y el religioso. Respecto a lo educativo, las mujeres refirieron que les hacía falta tener más información; ya que las escuelas carecen de educación sexual, en la mayoría de los casos la baja escolaridad dificulta asimilar algunos conceptos biomédicos o incluso poder leer sus resultados de la prueba de Papanicolaou. Una ausencia es que no mencionan la información de trípticos, carteles o anuncios del sector salud encaminados a la detección oportuna del CACU (Luna, Sánchez, 2014).

Un estudio realizado en Sinclejo, Colombia se encontró que el rango de edades tanto en las mujeres que se realizan la citología y las que no se la realizan oscila entre los 15 y 35 años. Tanto en las mujeres que se realizan la citología como en las que no se la realizan, la escolaridad estuvo representada por los grados de secundaria incompleta en un 33\% (52) y $31 \%$ (11) respectivamente; seguido de los grados secundaria completa en un $20 \%$ (31) y $16 \%$ (6) respectivamente. El estado civil predominante tanto en las mujeres que se realizan la citología como las que no se la realizan es la unión libre en un $55 \%$ (85) y $65 \%$ (24) respectivamente. Con relación a los conocimientos, se encontró que tanto las mujeres que se realizan la citología cérvico uterina como las que no se la realizan, tienen un conocimiento adecuado en un $95 \%$ (148) y un $92 \%$ (34) respectivamente y un conocimiento inadecuado de un 5\% (7) y del $8 \%$ (3). Entre las razones por las que las mujeres no se realizan la citología, se obtuvo razones como no considerarlo importante $75 \%$ (28), pena y embarazo en un $8 \%$ (3), no tener pareja en un 6\% (2) y miedo en un $3 \%$ (1) (Polo, Torres, Ochoa \& Villarreal, 2014).

Por lo analizado anteriormente se precisa la siguiente pregunta de investigación ¿cuáles son los factores socioculturales que intervienen para la realización de Papanicolaou en mujeres de la comunidad de Santo Tomas en el municipio de Tenosique, Tabasco? 


\section{Objetivo}

Identificar los factores socioculturales que influyen en la baja afluencia para la realización del Papanicolaou en mujeres de 25 a 35 años de la comunidad indígena de Santo Tomas en el municipio de Tenosique, Tabasco; de igual manera conocer el porcentaje de las mujeres que no se realizan el Papanicolaou y determinar si la aceptación de Papanicolaou por la pareja influiría en la aceptación de este estudio por las mujeres.

\section{Justificación}

La toma de la muestra de Papanicolaou, es el inicio de una serie de pasos en un proceso de detección, en la cual si no se realiza de acuerdo a la técnica se detiene diagnósticos erróneos como pueden ser resultados falsos positivos, negativas o no emitir un resultado las muestras se pueden clasificar como muestras satisfactorias, satisfactorias pero eliminadas y no satisfactorias o inadecuadas (Secretaria de Salud, 2006). La prueba de Papanicolaou fue introducida en 1943 por el Dr. George Papanicolaou como un examen de detección temprana para el cáncer cervical. Esta prueba está diseñada para la detección de lesiones del epitelio escamoso, aunque ha demostrado efectividad variable en la detección de otras malignidades que afectan el tracto genital femenino, debido a que el cérvix es fácilmente accesible durante el examen ginecológico y a que las lesiones cervicales se desarrollan lentamente en un periodo de aproximadamente 10 años, la prueba de detección de Papanicolaou ha demostrado ser muy efectiva (Marilin \& Amir, 2001).

Entre los requisitos principales para la realización de la prueba de Papanicolaou se encuentran: no estar en el período menstrual (regla), no haber tenido relaciones sexuales las 48 horas anteriores, no haberse realizado duchas vaginales en el lapso de 48 horas y no haberse aplicado ningún tratamiento médico vaginal (óvulos o cremas), durante las últimas 48 horas. La prueba de Papanicolaou no es dolorosa, mantener una actitud positiva será de mucha utilidad para que el examen transcurra sin ningún inconveniente, se recomienda estar relajada y sobre todo, eliminar los nervios. Todas las mujeres con 20 años o más, sin excepción, y las menores de 20 años que hayan mantenido relaciones sexuales deben realizarse ese procedimiento de detección. Entre los factores personales para la aplicación de un modelo de promoción de la salud, estos se clasifican en biológicos como: edad, sexo, índice de masa corporal, estado de pubertad, estado menopaúsico, la capacidad aeróbica, la fuerza y la agilidad; factores psicológicos se incluyen: autoestima, automotivación, competencia personal, el estado de salud percibido y la definición de salud; y factores socioculturales se incluyen la raza, etnicidad, aculturación, educación y estrato socioeconómico (Biblioteca Nacional de Salud y Seguridad Social Caja Costarricense de Seguro Social [BINASSS], 1996). 
En el 2012 se diagnosticaron 528,000 casos nuevos, y 266,000 mujeres murieron de esta enfermedad, casi el 90\% de ellas en países de ingresos bajos o medianos. Se prevé que, sin atención urgente, las defunciones debidas a cáncer cervicouterino podrían aumentar casi un 25\% durante los próximos 10 años (Organización Panamericana de la Salud [OPS] y Organización Mundial de la Salud [OMS], 2016).

En el año 2014 ocurrieron en el país 259,146 defunciones en mujeres mexicanas, de las cuales, los tumores malignos representaron el $14.6 \%$ de las muertes en mujeres $(38,046)$. Dentro de las neoplasias con mayor número de defunciones en mujeres se ubicaron el cáncer de mama y el CACU que en conjunto ocasionaron alrededor del $26 \%$ de todas las defunciones por cáncer en personas de este género. La tendencia de la mortalidad es descendente debido a una menor incidencia de la enfermedad por la mejora en las condiciones sociales y la respuesta de los sistemas de salud. Por lo tanto, constituye un indicador de desigualdad, ya que la mortalidad tiende a concentrarse a las regiones más desfavorecidas.

En México desde 2006 el cáncer de cuello uterino es la segunda causa de muerte por neoplasias en la mujer. Anualmente se estima una ocurrencia de 13,960 casos en mujeres, con una incidencia de 23.3 casos por 100,000 mujeres. En el año 2014, se registraron 3,063 casos nuevos de tumores malignos del cuello del uterino con una tasa de incidencia de 6.08 por 100,000 habitantes mayores de 10 años. En el grupo específico de mujeres de 25 años y más, se registraron 4,056 defunciones en mujeres con una tasa cruda de 11.9 defunciones por 100,000 mujeres y un promedio de edad a la defunción de 59.15 años. Las entidades con mayor mortalidad por CACU son: Chiapas (18.2) Morelos (17.2), Sonora (15.7), Quintana Roo (15.2), Chihuahua (15.1) y Baja California (15.0).

Uno de los factores altamente potenciales al CACU es el VPH; en el 2014 la tasa de positividad fue de 10.35, las entidades federativas que registraron el mayor índice de positividad fueron los estados de Veracruz, Tabasco y Chiapas con 13.1, 12.5 y 12.1 respectivamente. En relación a la clasificación por etapas clínicas de detección del CACU, el 23.27\% corresponden a carcinoma in situ, el $25.51 \%$ a etapas tempranas, el $16.61 \%$ a etapas intermedias, un $30.8 \%$ a etapas tardías y el $3.79 \%$ de los casos de CACU son tumores no clasificables.

Por lo tanto el CACU es un problema de salud pública mundial para las mujeres en edad reproductiva, al ubicarse como el segundo tipo de cáncer más común en mujeres. Actualmente, en Estados Unidos (EU) la incidencia de CACU ha disminuido a diferencia de países en desarrollo donde ha aumentado. El cáncer es un grupo de enfermedades caracterizadas por el crecimiento descontrolado y proliferación anormal de las células. De los diferentes tipos de cáncer que afectan a las mujeres, el Cérvico Uterino es una 
de las enfermedades más frecuentes (Bermúdez, 2005). Esta patología aparece en la unión escamocelular entre el epitelio columnar del endocervix y el epitelio escamoso de la ectocervix, donde ocurren los cambios metaplásicos.

El CACU se caracteriza por una etapa pre maligna (displasias) bien definida, la cual puede ser detectada mediante la examinación citológica. Debido al impacto positivo de la prueba de Papanicolaou, como método de tamizaje, es importante tanto para el patólogo como para el ginecólogo o médico primario, conocer los aspectos más importantes relacionados con este examen desde el momento de colección hasta su interpretación y seguimiento (Marilin y Amir, 2001). Se describen en el cuadro 1 las variables de estudio conceptual y operacional para ampliar el conocimiento y entender con mayor claridad, y así entender la problemática de salud que se vive hoy en día en la población femenina.

Cuadro 1. Definición de las variables de estudio

\begin{tabular}{|c|c|c|}
\hline Variable & Definición Conceptual & $\begin{array}{l}\text { Definición } \\
\text { Operacional }\end{array}$ \\
\hline Papanicolaou & $\begin{array}{l}\text { Es una prueba diagnóstica que } \\
\text { consiste en recoger una muestra } \\
\text { de células del cérvix o cuello del } \\
\text { útero y del canal cervical para } \\
\text { enviarlas al laboratorio y } \\
\text { someterlas a estudios (NOM- } \\
\text { 014-SSA2-1994). }\end{array}$ & $\begin{array}{l}\text { Cuestionario de } \\
\text { Actitudes hacia la } \\
\text { toma de } \\
\text { Papanicolaou por } \\
\text { Huamaní, Hurtado, } \\
\text { Guardia y Roca, } \\
\text { (2007) el cual es un }\end{array}$ \\
\hline Cáncer & Tumor maligno que inicia en el & instrumento que \\
\hline Cérvico & cuello de la matriz y es más & cuenta con \\
\hline Uterino & $\begin{array}{l}\text { frecuente en mujeres mayores de } \\
30 \text { años, al inicio las lesiones son } \\
\text { tan pequeñas que no se pueden } \\
\text { ver a simple vista y duran así } \\
\text { varios años (NOM-014-SSA2- } \\
\text { 1994). }\end{array}$ & $\begin{array}{l}\text { reactivos de los } \\
\text { cuales } 2 \text { son datos } \\
\text { generales y la } \\
\text { segunda parte valora } \\
\text { sobre actitudes hacia } \\
\text { la toma de }\end{array}$ \\
\hline $\begin{array}{l}\text { Factores } \\
\text { socioculturales }\end{array}$ & $\begin{array}{l}\text { Los factores sociales, son } \\
\text { aquellas cosas que afectan a los } \\
\text { seres humanos en su conjunto, } \\
\text { sea en el lugar y en el espacio en } \\
\text { el que se encuentren (Carrillo, } \\
\text { 2011). }\end{array}$ & $\begin{array}{lr}\text { Papanicolaou } & \text { con } 6 \\
\text { reactivos } & \text { con } \\
\text { opciones } & \text { de } \\
\text { respuesta con escala } \\
\text { de Likert. }\end{array}$ \\
\hline
\end{tabular}

\section{Metodología}

La investigación tiene un enfoque cuantitativo con diseño descriptivo de corte transversal; ya que buscábamos especificar las propiedades, las características y los perfiles de personas, grupo, comunidades, procesos, objetos o cualquier otro fenómeno que se someta a un análisis. Es útil para mostrar con precisión los ángulos o dimensiones de un fenómeno, suceso, comunidad, contexto o situación. 
Por otro lado fue una investigación prospectiva, por lo que se define como los diseños donde se reconstruyen las relaciones sobre la base de variabilidad amplia de las independientes y dependientes, no se parte de una variable en especial ni de grupos, sino que se evalúa la estructura causal completa (las relaciones en su conjunto). La población de estudio está integrada por 23 mujeres que no se realizan la detección del CACU; por lo cual, se realizó un muestreo probabilístico por conveniencia en donde las mujeres tienen edades entre 25 y 35 años con vida sexual activa. Se excluyeron a todas aquellas personas que no quisieron participar en la investigación y que se hayan realizado la detección en periodo menor a 12 meses.

Se utilizó el Cuestionario de Actitudes hacia la toma de Papanicolaou elaborado por Huamaní, Hurtado, Guardia y Roca, (2007), el cual es un instrumento que cuenta con 8 reactivos ( 2 del apartado de datos generales y 6 reactivos sobre actitudes hacia la toma de Papanicolaou); el instrumento presenta escala de medición tipo Likert. El instrumento de actitudes cuenta con opciones de respuestas de opción múltiple, se les dio un determinado tiempo aproximado de 30 minutos para responder y analizar las preguntas y respuestas esperamos junto a ellas mientras se respondía el cuestionario para resolver cualquier duda.

Se utilizó papel y lápiz para poder responder la encuesta a su vez la estudiante de la licenciatura en enfermería de la Universidad Juárez Autónoma de Tabasco (UJAT) en la División Académica Multidisciplinaria de los Ríos (DAMR) Francisca Velasco Zapata quien fue asesorada por profesores investigadores de la UJAT-DAMR.

Teniendo como fundamentos en investigación de enfermería cada una de las consideraciones éticas, se tomaron en cuenta las recomendaciones y consideraciones legales tanto la Ley General de Salud en Materia de Investigación para la Salud (Secretaria de Salud, 2016), el Código de Ética para Enfermeras y Enfermeros en México (CONAMED, 2015), la Declaración de Helsinki (Helsinki, 2015); Además de igual manera los requisitos mencionados en la NOM-012-SSA3-2012, que establece los criterios para la ejecución de proyectos de investigación para la salud en seres humanos (Diario Oficial de la Federación [DOF], 2013) donde se menciona la importancia de la firma del consentimiento informado, las características del individuo, el que se debe pugnar por el desarrollo de la profesión aplicando investigación científica, sin olvidar guarda la confidencialidad er intimidad del cliente sujeto de la investigación; de igual manera establecen que toda investigación debe ser aprobada por un comité de bioética y de investigación de la institución.

\section{Resultados}

De acuerdo a los datos obtenidos, el rango de edad más prevalente de entre aquellas mujeres en edad reproductiva que se rehúsan a realizarse la 
prueba se encuentran las ubicadas entre los 32 y 34 años de la comunidad de Santo Tomas siendo el $34.7 \%$ de la población, seguido por aquellas que se ubican en el rango de 29 y 31 años, posteriormente las ubicadas entre los 35 año y más y finalmente aquellas que se encuentran entre los 26 y 28 años (Ver tabla 1)

Tabla 1. Edades de mujeres indígenas en edad reproductiva.

\begin{tabular}{ccc}
\hline Edades & $f$ & $\%$ \\
\hline 26-28 años & 3 & 12.9 \\
29-31 años & 7 & 30.4 \\
32-34 años & 8 & 34.7 \\
35 años y mas & 5 & 21.7 \\
Total & 23 & 100.0 \\
\hline
\end{tabular}

En cuanto al nivel de escolaridad que presentaron las encuestadas predominó la educación primaria con un $43.5 \%$, seguido de las que se encuentran en nivel secundaria, preparatoria y aquellas que accedieron a educación superior (Ver tabla 2)

Tabla 2. Nivel de escolaridad

\begin{tabular}{ccc}
\hline Nivel de educación & $f$ & $\%$ \\
\hline Primaria & 10 & 43.5 \\
Secundaria & 9 & 39.1 \\
Preparatoria & 2 & 8.7 \\
Otros & 2 & 8.7 \\
Total & 23 & 100.0 \\
\hline
\end{tabular}

De igual manera al identificar el estado civil de las mujeres de la comunidad de Santo Tomas del municipio de Tenosique, Tabasco, se puede identificar que aquellas en el rubro de unión libre con 56.5\%, mientras que el $39.1 \%$ es casada y solo 1 es soltera (Ver tabla 3 ).

Tabla 3. Estado civil

\begin{tabular}{ccc}
\hline Estado civil & $f$ & $\%$ \\
\hline Soltera & 1 & 4.3 \\
Casada & 9 & 39.1 \\
Unión libre & 13 & 56.5 \\
Total & 23 & 100 \\
\hline
\end{tabular}

Aunado al estado civil se preguntó sobre el número de parejas sexuales donde, el $52.2 \%$ de las encuestadas ha tenido dos parejas sexuales, mientras que el $47.8 \%$ solo ha tenido una (Ver tabla 4).

Tabla 4.- Número de parejas sexuales

\begin{tabular}{ccc}
\hline No. de parejas & $f$ & $\%$ \\
\hline Uno & 11 & 47.8 \\
Dos & 12 & 52.2 \\
Total & 23 & 100.0 \\
\hline
\end{tabular}


Como información general las mujeres encuestadas saben cada cuanto tiempo deben de realizarse la prueba, y de igual manera saben cuál es la infección principal que causa el cáncer del cuello uterino con el 100\%. Pero de acuerdo a la información que reciben sobre el Papanicolaou respondieron sentirse de forma indiferente, y nunca se han realizado el Papanicolaou. Al realizar la encuesta donde se le pregunta saben para que se realiza la prueba del Papanicolaou las respuestas fueron incorrectas, de igual forma no saben en qué sitio del cuerpo se toma dicha muestra, pero respecto al momento de la vida en que se deben tomar la muestra de Papanicolaou las respuestas fueron correctas; es decir aunque afirmaron conocer la prueba, saben que el VPH es el principal precursor del CACU, pero no saben para que realizarla o porque existe esa necesidad de tomársela de forma periódica.

De igual manera un factor es el inicio de vida sexual activa, entre las mujeres encuestadas predominó el rango entre los 16-20 años con un 52.2\%, mientras que el $43.5 \%$ inicio a la edad de 15 o menos y por ultimo solo una se ubicó con una edad entre los 21 y 25 años (Ver tabla 5).

Tabla 5.- Edad de inicio de la vida sexual activa

\begin{tabular}{ccc}
\hline Inicio de Vida & $f$ & $\%$ \\
Sexual Activa & & 43.5 \\
15 o menos & 10 & 52.2 \\
$16-20$ años & 12 & 4.3 \\
$21-25$ años & 1 & 100.0 \\
Total & 23 & \\
\hline
\end{tabular}

Las encuestadas prefieren, al igual que sus parejas que el personal de salud que realice la citología debe ser de sexo femenino en el 100\%, pero al ser personas de comunidad las parejas de estas no les gusta que se realicen la prueba, es decir en el 100\% de los casos la pareja ha influido sobre la decisión de no acudir a realizarse la citología de forma oportuna, a pesar que el 100\% menciona que ha recibido buen trato del personal médico y enfermería.

\section{Discusión}

Los resultados obtenidos de este estudio demuestran que de un total de 23 mujeres el 100\% manifestó una actitud indiferente hacia la toma de Papanicolaou, en cuanto a la realización ninguna se lo ha realizado por motivos el desinterés, miedo al resultado y aunado a ello la opinión de sus parejas al no dejarlas que se realicen la prueba

También se obtuvo que el inicio de vida sexual activa predominante es entre los 16 y 20 años con un $52.2 \%$, y de este podemos decir que de acuerdo a Hernández (2010) el inicio de las relaciones sexuales en edades tempranas aumenta el riesgo de cáncer invasor de cuello uterino, considerándose factor determinante de las elevadas tasas de incidencia de cáncer cérvico uterino observadas en países en vías de desarrollo. 
En esta investigación las encuestadas prefieren, al igual que sus parejas que el personal de salud que realice la citología debe ser del sexo femenino, pero al ser personas de comunidad las parejas de estas no les gusta que se realicen la prueba lo cual coincide con el estudio realizado por Luna y Sánchez (2014) donde se asemejan las mismas situaciones con las parejas, pues manifiestan algunas conductas "machistas", como prohibir a sus parejas que acudan a revisión ginecológica porque "creen que la intimidad es de su propiedad". De igual manera en este trabajo hombre juega un papel importante en la patogénesis de esta enfermedad y es al que se le debería de dar énfasis en los programas preventivo promocionales, para sensibilizar a la población masculina sobre lo importante de prevenir la enfermedad invasiva de cuello uterino.

La educación sexual es básica para todas las personas que tienen acceso a la misma y en esta investigación el nivel de escolaridad que presentaron las mujeres en edad reproductiva de la comunidad de Santo Tomas predominó la educación primaria con un $43.5 \%$ y de acuerdo a la información que reciben sobre el Papanicolaou se manifiestan indiferente y nunca se han realizado el Papanicolaou, por lo que el desinterés en la detección oportuna a través de este procedimiento se considera también un factor de igual manera la religión juega un papel primordial donde $53.7 \%$ son mujeres con cultos religiosos presbiteriano o cristiano y $46.3 \%$ profesan la religión católica; lo que coincide con lo reportado por Luna y Sánchez (2014) donde ubicó como tercera esfera de importancia el nivel social, donde identificaron dos factores, el educativo y el religioso. Respecto a lo educativo, las mujeres refirieron que les hacía falta tener más información; ya que las escuelas carecen de educación sexual y que además su nivel educativo básico no favorecía el alcance de la información otorgada por el personal de salud.

La baja escolaridad dificulta asimilar algunos conceptos biomédicos o incluso poder leer sus resultados de la prueba de Papanicolaou y entenderlos; una ausencia es que no mencionan la información de trípticos, carteles o anuncios del sector salud encaminados a la detección oportuna del cáncer cervical en su comunidad lo que difiere con el estudio realizado por Polo, Torres, Ochoa \& Villarreal (2014) tanto en las mujeres que se realizan la citología como en las que no se la realizan, la escolaridad estuvo representada por los grados de secundaria incompleta y completa con un 33\% (52) y $31 \%$ (11) respectivamente; por lo que existe mayor nivel de escolaridad y por lo tanto se puede lograr una mayor comprensión de conceptos biomédicos.

Como se observa las similitudes y discrepancia entre los resultados de investigación realizadas en otros en otros países en vías de desarrollo e inclusive desarrollados dejan como resultado que existen factores como: la falta de educación sexual, la poca sensibilización de las parejas hombres y la pobre importancia que le dan a la prueba; lo que hace necesario que se requiere 
haga hincapié en la promoción de la realización de la prueba y que el sector salud sea participe de la sensibilización de las parejas hombres para que dejen a sus cónyuges participes de este proceso y que cada vez se les dé un diagnóstico oportuno mediante una detección temprana, lo que además permitirá minimizar las complicaciones en la salud de las mujeres se les dé un tratamiento adecuado y mejore la calidad de vida de la mujer y de su familia por el fuerte vínculo social de la mujer como pilar fundamental de la familia.

\section{Conclusión}

Existe incongruencia entre la información en las mujeres y su falta de interés en la realización de la prueba de Papanicolaou debido principalmente a la opinión negativa de parte del conyugue la cual juega un papel primordial en la decisión que toma la mujer en edad reproductiva de zonas rurales indígenas. Por lo anterior en necesario hacer énfasis en la importancia que actualmente han tomado los programas de salud que promueven las instituciones de salud gubernamentales; ya que hoy en día la mujer se muestra algo indispuesta a participar en la toma de Papanicolaou, puesto que influyen diversos factores como la pareja, creencias, etc., esto lo muestran los resultados obtenidos en el presente estudio.

Tal como se planteó en esta investigación, el CACU es una enfermedad prevenible, detectable $\mathrm{y}$, generalmente, curable si se diagnostica en sus estadios iniciales, pero para ello la información debe ser constante entre las mujeres y la promoción debe ser muy rigurosa para lograr dicha prevención. La disminución de su mortalidad a partir de la introducción del Papanicolaou es buena muestra de ello. Una tasa nacional elevada de mortalidad por CACU es no sólo un fracaso de los sistemas de salud sino una tragedia económica, familiar y afectiva que refrenda las evidencias de una inequidad social sobre todo en materia de atención sanitaria en las zonas rurales e inclusive indígenas.

\section{References:}

1. Secretaria de Salud (1994). NOM-014-SSA2-1994, para la prevención, tratamiento y control de cáncer del cuello del útero y de la mama en la atención primaria. Diario Oficial de la Federación. Recuperado

de: http://www.salud.gob.mx/unidades/cdi/nom/014ssa24.html

2. American Society of Clinical Oncology (ASCO, 2016). Prueba de Papanicolaou. Recuperado de: http://www.cancer.net/node/31891

3. Bembibre C (2009). Factor Sociocultural. Definición ABC. Recuperado de: https://www.definicionabc.com/social/sociocultural.ph

4. Luna-Blanco, M. A. \& Sánchez-Ramírez, G. (2014) Posibilidades sociales de prevención de la infección por virus del papiloma humano 
y de cáncer cervicouterino en San Cristóbal de Las Casas, Chiapas, México Revista Estudios Sociales y Humanísticos; 11 (2) 67-80. Recuperado de: http://www.redalyc.org/articulo.oa?id=74531037005

5. Polo Peña E. Y., Torres Pérez S.P. Ochoa Acosta R. \& Villarreal Amarís G. (2014) Factores Personales Relacionados con la Realización de Citología Vaginal Sincelejo 2013 - 2014. REVISALUD. Recuperado de: revistas.unisucre.edu.co/index.php/revisalud/article/download/124/12 5

6. Loustalot L. M., Espinoza R. R., Blas Rodríguez I., Vite Patiño G., Jerezano de la Rosa O. (2006) Manual de Procedimientos para la Toma de la Muestra de Citología Vaginal. Centro Nacional de Equidad de Género y Salud Reproductiva. Secretaria de Salud. Recuperado de: http://cnegsr.salud.gob.mx/contenidos/descargas/CaCu/toma_muestra .pdf

7. Marilin R. \& Amir M. (2001) Revisión Sobre Citología de Cuello Uterino con Énfasis en la clasificación de Bethesda. Departament of pathology. University of Louisville. Louisville, Estados Unidos de América. Recuperado de: http://www.conganat.org/9congreso/trabajo.asp?id_trabajo=794\&tipo $=1$

8. Biblioteca Nacional de Salud y Seguridad Social Caja Costarricense de Seguro Social (BINASSS, 1996). El Papanicolaou o Citología del Cuello Uterino. Recuperado de: http://www.binasss.sa.cr/poblacion/papanicolau.htm

9. Organización Panamericana de la Salud, Organización Mundial de la Salud. (2016). Control integral del cáncer cervicouterino. Guía de prácticas esenciales. Segunda edición. Recuperado de: http://iris.paho.org/xmlui/bitstream/handle/123456789/28512/978927 5318799_spa.pdf?ua=1

10. Centro Nacional de Equidad de Género y Salud Reproductiva (2016). Cáncer de Cuello Uterino. Secretaria de Salud. Recuperado de: http://cnegsr.salud.gob.mx/contenidos/Programas_de_Accion/Cancer delaMujer/CaCu/introduccion.html

11. Olivera Hernández M.T. (2015). Conocimientos, actitudes y practicas sobre la prueba de Papanicolaou en usuarias de la consulta externa de Gineco obstetricia del Hospital Alemán Nicaragüense en la Ciudad de Managua durante el periodo comprendido de octubre a diciembre del 2015. Repositorio Institucional UNAN-Managua [consulta 12 mayo 2017] disponible en: http://repositorio.unan.edu.ni/1456/1/38869.pdf

12. Asociación Médica mundial. (2015). Declaración de Helsinki: Recomendaciones para guiar a los médicos en la investigación 
biomédica en personas. Recuperado de: http://www.conamed.gob.mx/prof_salud/pdf/helsinki.pdf

13. Secretaria de Salud (2015). Reglamento de la Ley General de Salud en materia de investigación. Diario Oficial de la Federación. Recuperado de: www.salud.gob.mx/unidades/cdi/nom/compi/rlgsmis.html

14. Comisión Nacional de Arbitraje Médico (2003). Código de ética para enfermeras y enfermería en México. Recuperado de: http://www.conamed.gob.mx/prof_salud/pdf/codigo_enfermeras.pdf

15. Secretaria de Salud (2013). NOM-012-SSA3-2012, Que establece los criterios para la ejecución de proyectos de investigación para la salud en seres humanos. Diario Oficial de la Federación. Recuperado de: http://dof.gob.mx/nota_detalle.php?codigo $=5284148 \&$ fecha $=04 / 01 / 2$ 013

16. Hernández Alarcón R. (2014). Perfil epidemiológico y clínico de cáncer de cuello uterino- estado actual de la histerectomía radical en el servicio de ginecología del Hospital Edgardo Rebagliati Martins. Universidad Nacional Mayor de San Marcos. Sistema de Bibliotecas y Biblioteca Central. Recuperado de: http://sisbib.unmsm.edu.pe/bibvirtualdata/Tesis/Salud/hernandez_ar/ Cap2.pdf

17. Secretaría de Salud (2007). Manual de Consejería en Cáncer Cérvico Uterino. Recuperado

de: http://cnegsr.salud.gob.mx/contenidos/descargas/CaCu/manual_conse jeria.pdf 